\title{
Ácaros predadores (Acari) associados à cultura do morango (Fragaria sp, Rosaceae) e plantas próximas no Estado do Rio Grande do Sul
}

\author{
Noeli Juarez Ferla ${ }^{1,3}$, Marla Maria Marchetti ${ }^{2} \&$ Dinarte Gonçalves $^{2}$ \\ Biota Neotropica $v 7$ (n2) - http://www.biotaneotropica.org.br/v7n2/pt/abstract?article + bn01807022007 \\ Recebido em 10/11/06 \\ Versão reformulada recebida em 29/03/07 \\ Publicado em 01/05/07 \\ ${ }^{1}$ Pesquisador do Museu de Ciências Naturais, UNIVATES Centro Universitário, CP 155 \\ CEP 95900-000, Lajeado, Rio Grande do Sul, RS, Brazil \\ ${ }^{2}$ Mestrandos do Curso de Entomologia, Departamento de Biologia Animal, \\ Universidade Federal de Viçosa - UFV \\ ${ }^{3}$ Autor para correspondência: Noeli Juarez.Ferla, e-mail: njferla@univates.br
}

\begin{abstract}
Ferla, N.J., Marchetti, M.M. \& Gonçalves, D. Predatory mites (Acari) associated with strawberry and neighboring plants in the State of Rio Grande do Sul. Biota Neotrop. May/Aug 2007 vol. 7, no. 2 http://www.biotaneotropica.org.br/v7n2/pt/abstract?article+bn01807022007 ISSN 1676-0603.

The aim of this study was to determine the species predatory mites on strawberry crop and associated plants in the State of Rio Grande do Sul. It was conducted in Bom Princípio, Capitão and Lajeado counties, in crops of strawberry Oso Grande variety in low tunnels. The evaluations were done monthly between August 2002 and March 2004. In the plants of strawberry, the samples were constituted of three leaves collected in each of 15 plants taken randomly, totaling 45 leaves/field. In the associated plants, the sampling effort was of one hour for each plant evaluated. Mites of the families Ascidae, Parasitidae, Phytoseiidae, Cunaxidae, Erythraeidae, Stigmaeidae and Tydeidae were observed associated with the two-spotted spider mite, Tetranychus urticae Koch, 1836, important pest of this crop in the region where the study was conducted. Twenty species were identified, 14 of which belong to the family Phytoseiidae. Phytoseiulus macropilis (Banks, 1905) and Neoseiulus californicus (McGregor, 1954) were the most common species. On associated plants, the highest diversity of predatory mites was found on Richardia sp. (six mite species), Agerantum conyzoides L. (four mite species) and Sonchus oleraceus L., Bidens pilosa L. and Rumex sp. (three mite species). A dichotomous key is presented for the separation of
\end{abstract} the species collected.

Keywords: biodiversity, Phytoseiidae, Phytoseiulus macropilis, Neoseiulus californicus.

Resumo

Ferla, N.J., Marchetti, M.M. \& Gonçalves, D. Ácaros predadores (Acari) associados à cultura do morango (Fragaria sp, Rosaceae) e plantas próximas no Estado do Rio Grande do Sul. Biota Neotrop. May/Aug 2007 vol. 7, no. 2 http://www.biotaneotropica.org.br/v7n2/pt/abstract?article+bn01807022007 ISSN 1676-0603.

O objetivo deste estudo foi determinar as espécies de ácaros predadores associados à cultura do morango e em plantas associadas, no estado do Rio Grande do Sul. Este estudo foi conduzido nos municípios de Bom Princípio, Capitão e Lajeado em plantações de morango da variedade Oso Grande em túnel baixo. As avaliações foram feitas mensalmente entre agosto de 2002 e março de 2004. Nas plantas de morango, as amostras foram constituídas de três folhas coletadas em cada uma das 15 plantas escolhidas ao acaso, totalizando 45 folhas/campo. Nas plantas associadas, o esforço de amostragem foi de uma hora para cada planta avaliada. Ácaros das famílias Ascidae, Parasitidae, Phytoseiidae, Cunaxidae, Erythraeidae, Stigmaeidae e Tydeidae foram observados associados com o ácaro rajado, Tetranychus urticae Koch, 1836, praga importante na região onde este estudo foi conduzido. Vinte espécies foram identificadas, 14 das quais pertencem à família Phytoseiidae. Phytoseiulus macropilis (Banks, 1905) e Neoseiulus californicus (McGregor, 1954) foram as espécies mais comuns. Nas plantas asssociadas, a maior diversidade foi observada em Richardia sp. (seis espécies), Agerantum conyzoides L.(quatro espécies) e Sonchus oleraceus L., Bidens pilosa L.e Rumex sp. (três espécies). Uma chave docotômica é apresentada para a identificação das espécies.

Palavras-chave: biodiversidade, Phytoseiidae, Phytoseiulus macropilis, Neoseiulus californicus. 


\section{Introdução}

O morango (Fragaria sp.) é uma planta herbácea, rasteira e perene da família Rosaceae, propagada por via vegetativa, através de estolhos. Em geral, a cultura para produção de frutos é renovada anualmente. A parte comestível é um pseudo-fruto, originário do receptáculo floral que se torna carnoso e suculento. Seu cultivo é bastante desenvolvido em vários países do mundo, especialmente nos de clima temperado.

No Brasil, a cultura do morangueiro é uma importante atividade de famílias de pequenos e médios agricultores. No Estado do Rio Grande do Sul, embora cultivado há muitos anos, a partir da década de 1990 passou a ser de importância comercial, ocupando grande contingente de mão-de-obra, sendo de grande importância econômica e social. Normalmente é feita por produtores estabelecidos em minifúndios que utilizam à mão-de-obra familiar para a produção.

Doenças e pragas podem comprometer de forma significativa a produção desta cultura. Atualmente, os ácaros são os organismos que vêm causando maiores danos e prejuízos a esta cultura. Seu controle tem sido difícil, e o uso de produtos químicos para tanto pode causar problemas de contaminação (Garcia \& Chiavegato 1997).

Ácaros das famílias Tetranychidae são relatados como pragas importantes desta cultura. Dentre estes, o ácaro rajado (Tetranychus urticae Koch, 1836) é a espécie de maior relevância no Brasil e em diversas regiões produtoras de outros países. Ataca principalmente a face inferior das folhas desenvolvidas, provocando a formação de manchas branco-prateadas. Na face superior aparecem áreas inicialmente cloróticas, passando a bronzeadas, podendo haver seca e queda das folhas (Flechtmann 1979). Aparece principalmente na época de frutificação e colheita de frutos (Calza \& Suplicy 1967), podendo reduzir tanto a qualidade quanto a quantidade dos frutos (Chiavegato 1979).

$\mathrm{Na}$ forma convencional, o ácaro rajado é controlado com o uso de acaricidas. Esta forma de controle está sofrendo resistência dos consumidores devido à dificuldade em respeitar o período de carência e pela toxicidade dos produtos utilizados. Entretanto, vários estudos demonstram que ácaros predadores da família Phytoseiidae podem controlar, de forma efetiva, populações de ácaro rajado nesta cultura. Na Europa e Estados Unidos, Phytoseiulus persimilis Athias-Henriot demonstrou efetivo controle de T. urticae na cultura do morango (Simmonds 1971; Oatmann et al. 1976; Oatman et al. 1977a; Oatman \& McMurtry 1966). No Sul da Califórnia, Neoseiulus californicus (McGregor) tem sido liberado para o controle de tetraniquídeos em uma grande diversidade de culturas, incluindo morango (Strong \& Croft 1995; McMurtry \& Croft 1997), com efetivo controle da praga (Oatmann et al. 1977a, b). No Brasil, Phytoseiulus macropilis (Banks) é um fitoseídeo comumente encontrado associado a populações de tetraniquídeos, ocorrendo naturalmente em morangueiro nas regiões sul e sudeste (Garcia \& Chiavegato 1997, Marchetti \& Ferla 2004).

O objetivo deste estudo foi reconhecer as espécies de ácaros predadores em cultura de morangueiro e em plantas encontradas no interior ou nos arredores das culturas, no Estado do Rio Grande do Sul.

\section{Material e Método}

Este estudo foi conduzido nos municípios de Bom Princípio, Capitão e Lajeado, Rio Grande do Sul, em plantações de morango da variedade Oso Grande, mantidas em túnel baixo. As avaliações foram realizadas mensalmente entre agosto de 2002 e março de 2004.

Nas plantas de morango, as amostras foram constituídas de três folhas coletadas de cada uma das 15 plantas escolhidas ao acaso em cada campo, totalizando 45 folhas/campo. As folhas foram destacadas e postas separadamente em sacos plásticos, mantidas em caixa de isopor com gelo para manter baixa a temperatura, e levadas ao laboratório para realizar a contagem e coleta dos ácaros.
Ácaros predadores foram também coletados mensalmente em cinco espécies de plantas comumente encontradas no interior ou nos arredores da cultura de morango. Em cada uma das espécies o esforço amostral para coleta foi realizado durante um período de uma hora.

A contagem foi realizada diretamente sobre as folhas, utilizando microscópio estereoscópico, observando as duas faces da folha. Com a utilização de pincel de ponta fina, os ácaros foram coletados e guardados em álcool $70 \%$ para posterior montagem e identificação. Todos os ácaros foram montados em meio de Hoyer (Jeppson et al. 1975). As lâminas montadas foram mantidas em estufa a 50-60 ${ }^{\circ} \mathrm{C}$, por cerca de 10 dias para a fixação, distensão e clarificação dos espécimes e secagem do meio. A identificação foi feita com o auxílio de microscópio óptico com contraste de fases.

Espécimes representantes de cada uma das espécies encontradas foram depositados na Coleção de Referência de Ácaros do Museu de Ciências Naturais do Centro Universitário UNIVATES (ZAUMCN), Lajeado, Rio Grande do Sul.

\section{Resultados}

Foi encontrado um total de 855 ácaros pertencentes às famílias Ascidae, Cunaxidae, Erythraeidae, Parasitidae, Phytoseiidae, Stigmaeidae e Tydeidae, em 28 espécies de plantas analisadas. Vinte espécies de ácaros foram identificadas, 14 das quais pertencem à família Phytoseiidae. São apresentadas, a seguir, as espécies acarinas e as plantas sobre as quais as espécies acarinas foram encontradas.

\section{Ordem mesostigmata}

\section{ASCIDAE Voigts \& Oudemans, 1905}

\section{Lasioseius sp.}

Espécimes examinados: Capitão: Bidens pilosa L., XI-2002 (1); Richardia sp. III-2003 (1).

\section{PARASITIDAE Oudemans, 1901}

\section{Parasitus sp.}

Espécimes examinados: Capitão: Coronopus didymus (L.) Sm., XI-2003 (1).

\section{PHYTOSEIIDAE Berlese, 1913}

\section{Amblyseinae}

Amblydromalus limonicus (Garman \& McGregor, 1956)

Amblyseius limonicus Garman \& McGregor, 1956: 11; Moraes et al. 1994:211.

Amblyseiopsis limonicus Garman, 1958: 78.

Typhlodromus (Amblyseius) limonicus Chant, 1959:96.

Typhlodromus (Amblyseius) garmani (sinonímia, de acordo com Moraes et al. 1986).

Typhlodromalus limonicus DeLeon, 1967:22; Muma et al. 1970: 90.

Amblydromalus limonicus Chant \& McMurtry 2005: 203.

Espécimes examinados: Bom Princípio: S. oleraceus, VIII2003 (1).

Registros prévios no Brasil: Bahia, Minas Gerais e Pernambuco (Moraes et al. 1994).

\section{Amblyseius operculatus DeLeon, 1967}

Amblyseius operculatus DeLeon, 1967: 26; Denmark \& Muma, 1989: 47.

Espécimes examinados: Capitão: Rumex sp., VIII-2003 (1).

Registros prévios no Brasil: Pernambuco e São Paulo (Moraes et al. 2004). 


\section{Euseius concordis (Chant, 1959)}

Typhlodromus (Amblyseius) concordis Chant, 1959: 69. Amblyseius (Iphiseius) concordis Muma, 1961: 288.

Amblyseius concordis Chant \& Baker, 1965: 22; Moraes \& McMurtry, 1983: 138.

Euseius concordis Denmark \& Muma, 1973: 264; Feres \& Moraes, 1998: 127; Moraes \& McMurtry, 1983: 138; Ferla \& Moraes, 1998; Ferla \& Moraes, 2002a.

Espécimes examinados: Lajeado: Fragaria sp., II-2004 (4); III-2004 (5).

Registros prévios no Brasil: Bahia, Ceará, Minas Gerais, Paraíba, Pernambuco, Rio Grande do Sul e São Paulo (Moraes et al. 2004).

\section{Fundiseius sp.}

Espécimes examinados: Capitão: Fragaria sp., X-2003 (1).

\section{Neoseiulus anonymus (Chant \& Baker, 1965)}

Amblyseius anonymus Chant \& Baker, 1965: 21; McMurtry, 1983: 254; Schicha \& Elshafie, 1980: 32.

Neoseiulus anonymus Denmark \& Muma, 1972: 27; Kreiter \& Moraes, 1997: 378; Moraes \& Mesa, 1988: 76; Moraes et al., 1991: 126; Moraes et al., 2000: 245.

Espécimes examinados: Bom Princípio: Fragaria sp. IX-2002 (2); X-2002 (4). Lajeado: Fragaria sp., IX-2002 (2); X-2002 (4).

Registros prévios no Brasil: Mato Grosso (Ferla \& Moraes, 2002) Paraná, Pernambuco e São Paulo (Moraes et al. 2004).

\section{Neoseiulus californicus (McGregor, 1954)}

Typhlodromus californicus McGregor, 1954: 89; El-Banhawy, 1979: 113.

Neoseiulus chilenensis Dosse, 1958: 55 (sinonímia, de acordo com Athias-Henriot, 1977, El-Banhawy, 1979 e Moraes \& Mesa, 1988).

Espécimes examinados: Bom Princípio: Fragaria sp.IX-2002 (1); XI-2003 (7); X-2002 (9); XI-2002 (1); V-2003 (1); VII-2003 (1); IX-2003 (183); X-2003 (10). Capitão: Fragaria sp., X-2003 (2). Lajeado: Fragaria sp., XI-2003 (1); IX-2003 (7); IX-2002 (1); XI-2002 (2); XII-2002 (3); II-2003 (5); 9-II-2004 (2); Richardia sp., II-2003 (1).

Registros prévios no Brasil: Rio Grande do Sul (Ferla \& Moraes 1998 e 2002)

\section{Neoseiulus tunus (DeLeon, 1967)}

Typhlodromips tunus DeLeon, 1967: 29; Denmark \& Muma, 1973: 253;

Amblyseius tunus Feres \& Moraes, 1998: 126.

Neoseiulus tunus Ferla \& Moraes, 2002a: 872 e 2002b: 1018.

Espécimes examinados: Bom Princípio: Sonchus oleraceus L., IX-2002 (1). Capitão: S. oleraceus L., XI-2002 (1).

Registros prévios no Brasil: Rio Grande do Sul (Ferla \& Moraes 1998 e 2002b) e São Paulo (Moraes et al. 2004)

\section{Phytoseilus macropilis (Banks, 1905)}

Laelaps macropilis Banks, 1905: 139.

Phytoseiulus speyeri Evans, 1952: 398 (sinonímia de acordo com Kennet, 1958: 477).

Phytoseiulus chanti Ehara, 1966: 135 (sinonímia de acordo com Denmark \& Muma, 1973: 236).

Phytoseiulus macropilis Schuster \& Prichard, 1963: 279; Muma et al., 1970: 30; McMurtry, 1983: 259; Denmark \& Schicha, 1983: 31; Kreiter \& Moraes, 1997: 378.

Espécimes examinados: Bom Princípio: Ageratum conyzoides L., X-2002 (3); Fragaria sp., IX-2002 (187); X-2002 (9); V-2003 (1); VIII-2003 (3); IX-2003 (208); S. oleraceus, IX-2002 (2). Capitão:
Fragaria sp., XI-2002 (3); XI-2002 (3). Lajeado: Fragaria sp., XI-2003 (2)

Registros prévios no Brasil: Ceará, Minas Gerais, Rio Grande do Sul e São Paulo (Moraes et al. 2004).

\section{Proprioseiopsis cannaensis (Muma, 1962)}

Amblyseiulus cannaensis Muma, 1962: 4.

Amblyseius cannaensis Moraes \& McMurtry, 1983: 132.

Proprioseipsis cannaensis Muma et al. 1970: 38.

Espécimes examinados: Bom Princípio: Richardia sp., IV-2003

(1). Capitão: Fragaria sp., I-2003 (1).

Registros prévios no Brasil: Bahia, Maranhão, Pernambuco, Piauí e São Paulo (Moraes et al. 2004).

\section{Typhlodromalus aripo (DeLeon, 1967)}

Typhlodromalus aripo DeLeon, 1967:21; Denmark \& Muma, 1973: 258.

Amblyseius aripo Moraes \& McMurtry, 1983: 132; Moraes \& Mesa, 1988: 73; Feres \& Moraes, 1998: 126.

Espécimes examinados: Bom Princípio: A. conyzoides, X-2002 (2); X-2003 (2); XII-2002 (1); IV-2003 (7); XII-2003 (9); B. pilosa XII-2002 (5); I-2003 (3); IV-2003 (4); XII-2003 (1). Fragaria sp., IX-2002 (1); X-2002 (2); XI-2002 (3); III-2003 (3); VII-2003 (2); XII-2003 (1); III-2004 (10); Galinsoga sp., IV-2003 (6); Richardia sp., XII-2002 (4); IV-2003 (2); Rumex sp., IV-2003 (3); S. oleraceus X-2003 (5); I-2004 (2); Stachys arvensis L., II-2003 (10); XI-2003 (10). Capitão: B. pilosa I-2003 (1); II-2003 (2); Bowlesia incana Ruiz x Poa, VIII-2003 (1); Commelina sp., I-2003 (6); Fragaria sp., I-2003 (2); X-2003 (2); V-2003 (4); VIII-2003 (1); IX-2003 (4); XII-2003 (2); Galinsoga sp., XI-2002 (1); Richardia sp., III-2003 (1); Rumex sp. IV-2003 (1); Stachys arvensis L., VIII-2003 (1). Lajeado: B. pilosa X-2002 (1); Richardia sp., XII-2002 (4); IV-2003 (3).

Registros prévios no Brasil: Bahia, Ceará, Maranhão, Pernambuco, Piauí, Rio Grande do Sul e São Paulo (Moraes et al. 2004).

\section{Typhlodromips sp.}

Espécimes examinados: Bom Princípio: A. conyzoides XII-2002 (1); IV-2003 (1); Fragaria sp., X-2002 (1); X-2003 (1); XI-2002 (1); I-2004 (1); VII-2003 (1). Capitão: Fragaria sp., IX-2003 (1).

\section{Typhlodromips mangleae DeLeon, 1967}

Typhlodromips mangleae DeLeon, 1967: 28.

Amblyseius mangleae Moraes \& Mesa, 1988; Moraes et al. 1991: 124; Ferla \& Moraes, 2002: 1014.

Espécimes examinados: Bom princípio: A. conyzoides L., IV2003 (1); B. pilosa III-2003 (1); IV-2003 (3); Fragaria sp., III-2003 (1); Galinsoga sp., IV-2003 (2); Rumex sp., V-2003 (1); IV-2003 (1).

Registros prévios no Brasil: Pernambuco, Rio Grande do Sul e São Paulo (Moraes et al. 2004).

\section{Phytoseiinae}

\section{Phytoseius guianensis DeLeon, 1965}

Phytoseius guianensis DeLeon, 1965: 18; Denmark, 1966: 23; Denmark \& Muma, 1983: 144; Ferla \& Moraes, 2002b: 1019.

Espécimes examinados: Bom Princípio: Sida rhombifolia L., III-2003 (2). Vernonia polyanthes Lees. II-2003 (1).

Registros prévios no Brasil: Bahia, Ceará, Paraíba, Pernambuco, Piauí, Rio Grande do Sul e São Paulo (Moraes et al. 2004).

\section{Typhlodrominae}

1. Typhlodromus (Anthoseius) transvaalensis (Nesbitt, 1951)

Kampimodromus transvaalensis Nesbitt, 1951: 55. 
Typhlodromus transvaalensis Chant, 1955: 498; Ferla \& Moraes, 2002:1020.

Espécimes examinados: Capitão: Fragaria sp., VIII-2002 (1).

Registros prévios no Brasil: Rio Grande do Sul e São Paulo (Moraes et al. 2004).

\section{Ordem prostigmata}

Cunaxidae Thor, 1902

\section{Neocunaxoides sp.}

Espécimes examinados: Lajeado: Richardia sp. II-2003 (1).

\section{Erythraeidae}

\section{Callidosoma sp.}

Espécimes examinados: Lajeado: Fragaria sp., II-2003 (1); Richardia sp. II-2003 (1).
Stigmaeidae Oudemans, 1931

\section{Agistemus floridanus Gonzalez-Rodriguez, 1965}

Agistemus floridanus Gonzalez-Rodriguez, 1965: 42.

Espécimes examinados: Bom Princípio: Fragaria sp., X-2002 (2).

\section{Tydeidae Kramer, 1877}

\section{Pronematus sp.}

Espécimes examinados: Bom Princípio: Fragaria sp., VIII2002 (1); XI-2002 (1); Leonurus sibiricus L., X-2003 (1); Capitão: Fragaria sp., III-2003 (1).

Os ácaros encontrados em cada uma das espécies de plantas amostradas são apresentados na Tabela 1.

A chave dicotômica apresentada a seguir pode ser utilizada para a separação dos grupos de ácaros encontrados.

1. Tarso do palpo com apotele; tritosterno presente; estigmas localizados lateralmente entre as coxas III e IV e associados ao peritrema . Ordem Mesostigmata................... 2

- Tarso do palpo sem apotele; tritosterno ausente; sem estigmas no segundo par de coxas. Ordem Prostigamata ................. 17

2. Escudo genital triangular e ladeado por escudos metaesternais desenvolvidos. Apotele trifurcado

Parasitidae.

- Escudos genitais e metaesternais não como acima. Apotele bifurcado

Com mais de 24 pares de setas dorsais Ascidae Lasioseius sp.

- Com menos de 24 pares de setas dorsais.

4. Região podonotal do escudo dorsal com quatro pares de setas "laterais" (j3, z2, z4 e s4)

Amblyseiinae 6

- Região podonotal do escudo dorsal com seis pares de setas "laterais" ( $j 3, z 2, z 3, z 4$, s4 e s6)

5. Seta $Z 1, S 2, S 4$ e $S 5$ ausentes

Phytoseinae Phytoseius guianensis

- Ao menos uma das setas Z1, S2, S4 e $S 5$ presentes Typhlodrominae Typhlodromus transvaalensis

6. Setas j6 mais longa que o dobro da distância entre suas bases. Phytoseiulus macropilis - Setas j6 menores que o dobro da distância entre suas bases

7. Seta $J v 1$ afastada da margem anterior do escudo ventrianal; peritrema normalmente curto, raramente estendendo-se além da base da seta $z 2$

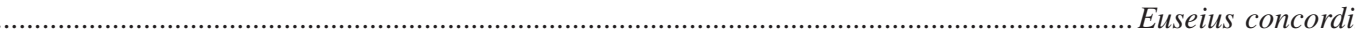
- Seta $J v 1$ inserida próxima à margem do escudo ventrianal; peritrema normalmente estendendo-se até a base da seta $j 1 \ldots \ldots \ldots \ldots \ldots \ldots \ldots . . . . .8$

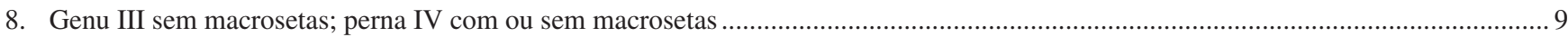

- Genu III e genu, tíbia e tarso IV com macrosetas (às vezes outras pernas também com macrosetas ................................................. 12

9. Fortemente esclerotizados; marrons; escudo ventrais relativamente amplos; perna IV com macrosetas ..... Fundiseius - Levemente esclerotizados; coloração levemente amarelada; escudos ventrais pouco alargados; perna IV com ou sem macrosetas.........

10. Maioria das setas do escudo dorsal longas, geralmente ultrapassando a distância entre as bases das setas vizinhas .

- Maioria das setas do escudo dorsal curtas, não alcançando as bases das setas vizinhas.

Neoseiulus anonymus

1. Algumas setas da metade anterior do escudo dorsal serreadas; escudo dorsal com estriações leves..... Neoseiulus tunus - Todas as setas da metade anterior do escudo dorsal lisas; escudo dorsal sem estrias ou com estriações bem pronunciadas .. Neoseiulus californicus

12. Seta $J 2$ ausente.... Proprioseiopsis cannaensis - Seta $J 2$ presente 13

13. Tarso I com seta proximal ereta; setas $Z 5$ consideravelmente maiores que a distância entre as suas bases...Amblyseius operculatus - Tarso I sem seta proximal ereta; setas Z5 normalmente menores que a distância entre as suas bases 
14. Margem posterior do escudo esternal trilobada, frequentemente não visível; escudo ventrianal em forma de vaso .............................. 1 - Margem posterior do escudo esternal reta ou côncava, sempre bem visível; escudo ventrianal aproximadamente pentagonal ............. 16

15. Razão do comprimento entre as setas $s 4$ : Z1 é maior do que 3:1; escudo dorsal liso; setas Z4 com $13 \mu$ m........Amblydromalus limonicus - Razão do comprimento entre as setas s4: Z1 é menor do que 3:1; escudo dorsal reticulado; setas Z4 com $67 \mu \mathrm{m}$.

Typhlodromalus aripo

16. Setas $s 4$ com $23 \mu \mathrm{m}, Z 4$ com $33 \mu \mathrm{m}$ e Z5 com $69 \mu \mathrm{m}$. Typhlodromips mangleae - Setas $s 4$ com $58 \mu \mathrm{m}, Z 4$ com $68 \mu \mathrm{m}$ e Z5 com $70 \mu \mathrm{m}$. Typhlodromips

17. Quelas edentadas ou em lâmina recurvada; com dois pares de tricobótrias no dorso do propodossoma............................................. 18 - Quelas em forma de estiletes; com um ou nenhum par de tricobótrias no dorso do propodossoma ......

18. Adultos e ninfas com corpo densamente coberto de setas; dígito móvel da quelícera longo e retilíneo; sem discos genitais.

Erythraeidae.......

..Callidossoma

- Adultos e ninfas com corpo com poucas setas; dígito móvel em forma de lâmina recurvada; com discos genitais Cunaxidae

Neocunaxoides

19. Com um par de tricobótrias no dorso do propodossoma; sem escudos dorsais, tarso I sem apotele; sem seta no trocanter I .........Tydeidae

- Sem tricobótrias no dorso do propodossoma; com escudos dorsais; tarso I com apotele Stigmaeidae .Agistemus floridanus

\section{Discussão}

Neste estudo, os fitoseídeos foram os ácaros que apresentaram maior diversidade, estando presentes na maioria das plantas amostradas (Tabela 1). Dos 855 ácaros coletados, 843 pertenceram a esta família. Quatorze espécies, pertencentes a nove gêneros estiveram representadas, sendo $P$. macropilis e $N$. californicus as espécies mais abundantes. Nesta família, o gênero Neoseiulus Hughes apresentou três espécies, seguidos do gênero Typhlodromips DeLeon, com duas espécies. As demais famílias acarinas (Ascidae, Cunaxidae, Erythraeidae, Parasitidae, Stigmaeidae e Tydeidae) foram encontradas apenas esporadicamente.

Phytoseiulus macropilis e $N$. californicus com 421 e 237 espécimes, respectivamente, foram coletados, em sua maioria, sobre Fragaria sp.. Typhlodromalus aripo, com 135 espécimes esteve presente sobre Fragaria sp. e demais plantas avaliadas.

Agistemus floridanus foi encontrado apenas em Fragaria sp.. Esta espécie foi encontrada em abundância em plantações de seringueira no estado do Mato Grosso (Ferla \& Moraes 2002). Apresenta altos níveis de oviposição quando alimentado com Tetranychus mexicanus (McGregor) (Moraes \& Ferla 2003), indicando que este ácaro possa se manter no ambiente alimentando-se de T. urticae. Entretanto, devido ao baixo número de espécimes neste estudo, T. urticae parece não ser presa adequada. Mattioli \& Oliveira (2000) observaram maior oviposição de Agistemus aff. bakeri quando alimentado com B. phoenicis se comparado a T. urticae e Panonychus citri (McGregor).

Em relação à espécie vegetal sobre a qual cada ácaro foi encontrado, Typhlodromalus aripo e Typhlodromips mangleae foram as espécies de maior freqüência, tendo sido encontradas em dez e cinco espécies, respectivamente, das 28 espécies vegetais avaliadas. Typhlodromalus aripo é uma espécie de ácaro predador comumente associada a Mononychellus tananjoa (Bondar) na cultura de mandioca no Nordeste (Moraes et al. 1990), encontrado quase que exclusivamente nos brotos terminais (Bakker 1993). Também foi relatado em outras culturas e em plantas daninhas em associação com outras espécies acarinas (Moraes \& McMurtry 1983; Moraes et al. 1990; Noronha et al. 1997). Typhlodromips mangleae também foi observado, porém em menor número, em plantas nativas no estado do Rio Grande do Sul (Ferla \& Moraes 2002).

Quanto às plantas invasoras encontradas nas proximidades das campos de morangueiro, Richardia sp., Agerantum conyzoides, Sonchus oleraceus, Bidens pilosa e Rumex sp. foram as que apre- sentaram maior número de espécies de ácaros com seis, quatro, quatro e três espécies, respectivamente. Algumas espécies de plantas apresentaram baixa diversidade de predadores e em catorze espécies vegetais não foram encontrados ácaros predadores. Estes resultados, entretanto, podem estar influenciados pela freqüência com que cada espécie vegetal foi encontrada nos diferentes locais avaliados por este estudo, de forma que a ausência ou baixa freqüência de predadores em determinada espécie vegetal pode ser devido ao fato de que poucas plantas desta espécie tenham sido avaliadas. Assim, a maior diversidade e abundância em Fragaria sp. pode ser explicada pelo maior esforço amostral e maior abundância nos locais avaliados.

As frequiências relativas das famílias encontradas e os níveis de diversidade dentro de cada família estão compatíveis com o que seria esperado de acordo com os resultados de outros autores em outras regiões (Ferla \& Moraes 2002). Os fitoseídeos são, de maneira geral, os predadores mais abundantes e diversos em plantas cultivadas e silvestres, seguidos, normalmente, pelos estigmeídeos, numa distante segunda posição. Ácaros destas duas famílias têm sido citados preponderantemente como predadores de outros ácaros (Moraes 2002; Ferla \& Moraes 1998). Outros grupos de ácaros predadores também encontrados neste estudo parecem ter alimentação mais diversa, incluindo vários insetos como presas, e usualmente ocorrem em níveis muito menores (Gerson 1985; Moraes 2002). Ferla \& Moraes (2002) avaliaram a diversidade de ácaros predadores plantícolas em vegetação nativa e introduzida no Rio Grande do Sul e encontraram 30 espécies de ácaros predadores, sendo Iphiseiodes zuluagai Denmark \& Muma a mais abundante. Naquele estudo, T. aripo e N. californicus também estiveram presentes, porém em menores proporções que as encontradas no presente trabalho.

Muitos trabalhos têm enfatizado o uso de culturas de cobertura em pomares para o aumento da população de inimigos naturais, voltados em sua maioria para o controle biológico conservativo. $\mathrm{Na}$ família Phytoseiidae, os ácaros generalistas se alimentam de pólen, fungos e ácaros e podem sobreviver em pomares em alta densidade mesmo quando as presas primárias são escassas ou temporariamente extintas, enquanto que as espécies especialistas necessitam que uma determinada espécie de presa permaneça no ambiente, sendo que na sua ausência se extinguem localmente por falta de alimento (Altieri 2003, McMurtry \& Croft 1997, Moraes 1991). Dentre as espécies encontradas, apenas $P$. macropilis, observado principalmente sobre Fragaria sp., pertenceu a categoria dos fitoseídeos predadores espe- 
Tabela 1. Ácaros predadores associados à cultura de morango e plantas próximas nos municípios de Bom Princípio, Capitão e Lajeado, Rio Grande do Sul. Table 1. Predatory mites associated with strawberry and neighboring plants in Bom Princípio, Capitão e Lajeado counties, State of Rio Grande do Sul.

\begin{tabular}{|c|c|c|c|}
\hline Família & Espécie vegetal & Espécie acarina & Espécimes \\
\hline AMARANTHACEAE & Amaranthus lividus & - & - \\
\hline \multirow[t]{16}{*}{ ASTERACEAE } & Ageratum conyzoides & Phytoseiulus macropilis & 3 \\
\hline & & Typhlodromalus aripo & 21 \\
\hline & & Typhlodromips sp. & 2 \\
\hline & & Typhlodromips mangleae & 1 \\
\hline & Bidens pilosa & Lasioseius sp. & 1 \\
\hline & & Typhlodromalus aripo & 17 \\
\hline & & Typhlodromips mangleae & 4 \\
\hline & Galinsoga sp. & Typhlodromalus aripo & 7 \\
\hline & & Typhlodromips mangleae & 2 \\
\hline & Jaegeria hirta & - & - \\
\hline & Sonchus oleraceus & Amblydromalus limonicus & 1 \\
\hline & & Neoseiulus tunus & 2 \\
\hline & & Phytoseiulus macropilis & 2 \\
\hline & & Typhlodromalus aripo & 7 \\
\hline & Synechellopsis grisebachii & - & - \\
\hline & Veronia polyanthes & Phytoseius guianensis & 1 \\
\hline \multirow[t]{2}{*}{ BRASSICACEAE } & Coronopus didymus & Parasitus sp. & 1 \\
\hline & Raphanus sativus & - & - \\
\hline CARYOPHYLLACEAE & Stellaria media & - & - \\
\hline COMMELINACEAE & Commelina sp. & Typhlodromalus aripo & 6 \\
\hline CONVOLVULACEAE & Ipomoea $\mathrm{sp}$. & - & - \\
\hline \multirow[t]{2}{*}{ LAMIACEAE } & Leonurus sibiricus & Pronematus sp. & 1 \\
\hline & Stachys arvensis & Typhlodromalus aripo & 21 \\
\hline \multirow[t]{2}{*}{ MALVACEAE } & Sida rhombifolia & Phytoseius guianensis & 2 \\
\hline & Sida santaremnensis & - & - \\
\hline OXALIDACEAE & Oxalis corniculata & - & - \\
\hline \multirow[t]{2}{*}{ POACEAE } & Brachiaria sp. & - & - \\
\hline & Lolium multiflorum & - & - \\
\hline \multirow[t]{3}{*}{ POLYGONACEAE } & Rumex sp. & Amblyseius operculatus & 1 \\
\hline & & Typhlodromalus aripo & 4 \\
\hline & & Typhlodromips mangleae & 2 \\
\hline \multirow[t]{13}{*}{ ROSACEAE } & Fragaria sp. & Euseius concordis & 9 \\
\hline & & Fundiseius sp. & 1 \\
\hline & & Neuseiulus anonymus & 12 \\
\hline & & Neoseiulus californicus & 236 \\
\hline & & Phytoseilus macropilis & 416 \\
\hline & & Proprioseipsis cannaensis & 1 \\
\hline & & Typhlodromalus aripo & 37 \\
\hline & & Typhlodromips sp. & 6 \\
\hline & & Typhlodromips mangleae & 1 \\
\hline & & Typhlodromus transvaalensis & 1 \\
\hline & & Callidosomo sp. & 1 \\
\hline & & Agistemus floridanus & 2 \\
\hline & & Pronematus sp. & 3 \\
\hline \multirow[t]{5}{*}{ RUBIACEAE } & Conyza canadensis & - & - \\
\hline & Richardia sp. & Neocunaxoides sp. & 1 \\
\hline & & Callidosoma sp. & 1 \\
\hline & & Propriseiopsis cannaensis & 1 \\
\hline & & Lasioseius sp. & 1 \\
\hline
\end{tabular}


Tabela 1. Continuação...

\begin{tabular}{|c|c|c|c|}
\hline Família & Espécie vegetal & Espécie acarina & Espécimes \\
\hline \multirow[t]{2}{*}{ RUBIACEAE } & Richardia sp. & Neoseiulus californicus & 1 \\
\hline & & Typhlodromalus aripo & 14 \\
\hline SCROPHUARIACEAE & Veronica persica & - & - \\
\hline \multirow[t]{2}{*}{ SOLANACEAE } & Solanum americanum & - & - \\
\hline & Solanum evanthum & - & - \\
\hline UMBELLIFERAE & Bowlesia incana & Typhlodromalus aripo & 1 \\
\hline Total de ácaros & & & 855 \\
\hline
\end{tabular}

- espécies vegetais onde não foram encontrados ácaros predadores.

cialistas. Além disso, $N$. californicus e $N$. anonymus são predadores seletivos que também podem se alimentar na presença de tetraniquídeos. As demais espécies são generalistas, sendo que muitas delas carecem de estudos ecológicos que definam a presa mais adequada e sua capacidade de controle de pragas.

\section{Referências Bibliográficas}

ALTIERI, M. A.; SILVA E. N. \& NICHOLLS, C. I. 2003. O papel da biodiversidade no manejo de pragas, Holos. Ribeirão Preto. p.226.

ATHIAS-HENRIOT, C. 1977. Nouvelles notes sur les Amblyseiini. III. Sur le genre Cydnodromus: Redefinition, composition (Parasitifomes, Phytoseiidae). Entomophaga, France, 22, 61-73.

BANKS, N. 1905. Descriptions of some new mites. Proceedings of the Entomological Society of Washington, USA, 7, 133-142.

BAKKER, F.M. 1993. Selecting phytoseiid predators for biological control, with emphasis on the significance of tritrophic interactions. Amsterdam, 131p. Thesis - University of Amsterddam.

CALZA, R. \& SUPLICY, N. 1967. Estudos sobre o "ácaro do morangueiro" Tetranychus telarius (L.). Biológico. 33:137-143.

CHANT, D.A. 1955. Notes on mites of the genus Typhlodromus Scheuten, 1857 (Acarina: Laelaptidae), with descriptions of the males of some species and the female of a new species. The Canadian Entomologist, Canada, 87(11), 496-503.

CHANT, D.A. 1959. Phytoseiid mites (Acarina: Phytoseiidae). Part I. Bionomics of seven species in southeastern England. Part II. A taxonomic review of the family Phytoseiidae, with descriptions of thirty-eight new species. The Canadian Entomologist, Ottawa, 91(12):1-166.

CHANT, D.A. \& BAKER, E.W. 1965. The Phytoseiidade (Acarina) of Central America. Memoirs of the Entomological Society of Canada, Ontario, 41:1-56.

CHANT, D.A. \& MCMURTRY, J.A. 2005. A review of the subfamily Amblyseiinae Muma (Acari: Phytoseiidae): Part VI. The tribe Euseiini N. Tribe, Subtribes Typhlodromalina N. subtribe, Euseiina N. Subtribe, and Ricoseiina N. subtribe. International Journal of Acarology, 31(3):187-224.

CHIAVEGATO, L.G. 1979. O ácaro Tetranychus (Tetranychus) urticae (Koch, 1836) - Boudreaux \& Dosse, 1963 (Acari: Tetranychidae) na cultura do morangueiro: subsídios para estudos de resistência e efeitos do seu ataque no cultivar "Campinas". Botucatu, 1979. 139p, Tese (Livre Docência) - FCA/UNESP.

DELEON, D. 1965. Phytoseiid mites from Puerto Rico with descriptions of new species (Acarina: Mesostigmata). Florida Entomologist, Gainesville, 48(2):121-131.

DELEON, D. 1967. Some mites of the Caribbean Area. Part I. Acarina on plants in Trinidad, West Indies. Kansas, Allen Press, 26p.

DENMARK, H. A. 1966. Revision of the genus Phytoseius Ribaga, 1904 (Acari: Phytoseiidae). Florida Department of Agriculture Bulletin, USA, $6,1-105$.
DENMARK, H.A. \& MUMA, M.H. 1972. Some Phytoseiidae of Colombia (Acarina: Phytoseiidae). Florida Entomologist, Gainesville, 55(1):19-29.

DENMARK, H.A. \& MUMA, M.H. 1973. Phytoseiid mites of Brazil (Acarina: Phytoseiidae). Revista Brasileira de Biologia, São Carlos, 33:235-276.

DENMARK, H.A. \& MUMA, M.H. 1989. A revision of the genus Amblyseius Berlese, 1914 (Acari: Phytoseiidae). Occasional Paper of the Florida State Collection of Arthropods, Gainesville, 4:1-149.

DENMARK, H. A. \& SCHICHA, E. 1983. Revision of the genus Phytoseiulus Evans (Acarina: Phytoseiidae). International Journal of Acarology, USA, 9, 27-35.

DOSSE, G. 1958. Uber einige neue Raubmilbenarten (Acar., Phytoseiidae). Pflanzenschutzberichte 21:44-61.

EHARA, S. (1966a) A tentative catalogue of predatory mites of Phytoseiidae Known from Asia, with descriptions of five species from Japan. Mushi, Japan, 39, 9-30.

EL-BANHAWY, E.M. 1976. A new predacious mite of the genus Typhlodromus Scheuten from Brazil. Revista Brasileira de Biologia, São Carlos, 36(2):531-534.

EL-BANHAWY, E.M. 1979. Records on phytoseiid (Acari) mites of Peru. International Journal of Acarology, USA, 5(2), 111-116.

EVANS, G.O. (1952a) A new typhlodromid mites predaceous on Tetranychus bimaculatus Harvey in Indonesia, Annual Magazine of Natural History, London, 5, 413-416.

FERES, R.J.F. \& MORAES, G.J. de. 1998. Phytoseidae mites (Acari: Phytoseiidae) from woody areas in the State of São Paulo, Brazil. Systematic and Applied Acarology, Amsterdam, 3:125-132.

FERLA, N.J. \& MORAES, G.J. de. 2002a. Ácaros (Arachnida, Acari) da seringueira (Hevea brasiliensis Muell. Arg.) no Estado do Mato Grosso. Revista Brasileira de Zoologia, Curitiba, 19(3):867-888.

FERLA, N.J. \& MORAES, G.J. de. 2002b. Ácaros predadores (acari) em plantas nativas e cultivadas do Estado do Rio Grande do Sul, Brasil. Revista Brasileira de Zoologia, Curitiba, 19(4):1011-1031.

FERLA N.J. \& MORAES, J.G. de 2003. Oviposição dos ácaros predadores Agistemus floridanus Gonzalez, Euseius concordis (Chant) e Neoseiulus anonymus (Chant \& Baker) (Acari) em resposta a diferentes tipos de alimento. Revista Brasileira de Zoologia, Curitiba, v. 20, n. 1, p.153-155.

FERLA, N.J. \& MORAES, G.J. de. 1998. Ácaros predadores em pomares de maçã no Rio Grande do Sul. Anais da Sociedade Entomológica do Brasil, Londrina, 27(4):649-654.

FLECHTMANN, C.H.W. 1979. Ácaros de importância agrícola. São Paulo, Livraria Nobel, 189p.

GARCIA, I.P. \& CHIAVEGATO, L.G. 1997. Resposta funcional e reprodutiva de Phytoseiulus macropilis (Banks, 1905) (Acari: Phytoseiidae) a diferentes densidades de ovos de Tetranychus urticae (Koch, 1836) (Acari: Tetranychidae). Científica, São Paulo, 25(1):35-43. 
GARMAN, P. (1958). New species belonging to the genera Amblyseius and Amblyseiopsis with keys to Amblyseius, Amblyseiopsis, and Phytoseiulus. Annals of the Entomological Society of America, USA, 51, 69-79.

GARMAN, P. \& McGREGOR, E.A. (1956). Four new predaceous mites (Acarina: Phytoseiidae). Southern California Academy of science Bulletin, USA, 55, 7-13.

GERSON, U. (1985). Webbing. In Spider Mites: Their Biology, Natural Enemies and Control, (eds W. Helle, and M. W. Sabelis), Elsevier, Amsterdam, Vol. 1A, pp. 223-32.

JEPPSON, L.R.; KEIFER, H.H. \& BAKER, E.W. 1975. Mites injurious to economic plants. Berkeley: University of California Press, 614p.

KENNETT, C.E. (1958). Some predacious mites of the subfamilies Phytoseiinae and Aceosejinae (Acari: Phytoseiidae, Aceosejidae) from central California with descriptions of new species. Annals of the Entomological Society of America, USA, 51, 471-479.

KREITER, S. \& MORAES, G.J. de. 1997. Phytoseiid mites (Acari: Phytoseiidae) from Guadalupe and Martinique. Florida Entomologist, Gainesville, 80(3):376-382.

MARCHETTI, M.M \& FERLA, N.J. 2004. Flutuação populacional de ácaros (Acari) na cultura do morango no Estado do Rio Grande do Sul. In: CONGRESSO BRASILEIRO DE ZOOLOGIA, 25., 2004, Brasília. Resumos..., Brasília: Sociedade Brasileira de Zoologia, 1994. p.15.

MATIOLI, A.L.; UECKERMANN, E.A. \& OLIVEIRA, C.A.L. 2002. Some stigmaeid and eupalopsellid mites from citrus orchards in Brazil (Acari: Stigmaeidae and Eupalopsellidae). International Journal of Acarology, USA, Vol. 28, No. 2, 99-120.

McGREGOR, E. A. (1954). Two new species in the genus Typhlodromus (Acari: Phytoseiidae). Southern California Academy os Science Bulletin, USA, 53, 89-92.

McMURTRY, J.A. (1983). Phytoseiid mites from Guatemala, with descriptions of two new species and redefinitions of the genera Euseius, Typhloseiopsis, and the Typhlodromus occidentalis species group (Acari: Mesostigmata). International Journal of Entomology, Honolulu, Hawaii, 25, 249-272.

McMURTRY, J.A. \& CROFT, B.A. 1997. Life-styles of phytoseiid mites and their roles in biological control. Annual Review of Entomology, Palo Alto, 42:291-321.

MORAES, G.J. de. 1991. Controle biológico de ácaros fitófagos. Inf. Agropec. 15:55-62.

MORAES, G.J. de. 2002. Controle biológico de ácaros fitófagos com ácaros predadores. In. CONTROLE BIOLÓGICO NA BRASIL: parasitóides e predadores. São Paulo. Manole, 2002. 635 p.: il.

MORAES, G.J. de \& MCMURTRY, J.A. 1983. Phytoseiid mites (Acarina) of northeastern Brazil, with descriptions of four new species. International Journal of Acarology, West Bloomfield, 9(3):131-148.

MORAES, G.J. de \& MESA, N.C. 1988. Mites of the family Phytoseiidae (Acari) in Colombia, with descriptions of three new species. International Journal of Acarology, West Bloomfield, 14(2):71-88.

MORAES, G.J. de; MESA, N.C. \& BRAUN, A.1991. Some phytoseiid mites of Latin America (Acari: Phytoseiidae). International Journal of Acarology, West Bloomfield, 17(2):117-139.

MORAES, G. J. de; DENMARK, H.A. \& MCMURTRY, J.A.1986. A catalog of the mite family Phytoseiidae: references to taxonomy, synonymy, distribution and habitat. Brasília, EMBRAPA-DDT, 353p.

MORAES, G.J. de; BRAUN, A.R.; YANINEK, J.S.; ALENCAR, J. A. 1990. Preliminary selection of predators of the cassava green mite (Acari: Tetranychidae) in Brazil for introduction into Africa. In: INTERNATIONAL CONGRESS OF ACAROLOGY, 8. 1990. Ceske Budejovice, Czechoslovalia. Abstracts. Hague: SPB Academic Publisching, 1990. p.108.

MORAES, G.J. de,; MESA, N.C., BRAUN, A. \& MELO, E.L. 1994. Definition of the Amblyseius limonicus species group (Acari: Phytoseiidae), with descriptions of two new species and new records. International Journal of Acarology, USA, 20(3), 209-217.
MORAES, G.J. de,; KREITER, S. \& LOFEGO, A.C. (2000) (1999). Plant mites (Acari) of the French Antilles. 3. Phytoseiidae (Gamasida). Acarologia, France, 40(3), 237-264.

MORAES, G.J. de,; McMURTRY, J.A.; DENMARCK, H.A. \& CAMPOS C.B. 2004. A revised catalog of the mites family Phytoseiidae. (Zootaxa 434), $494 \mathrm{p}$.

MUMA, M.H. 1961. Subfamilies, genera, and species of Phytoseiidae (Acarina: Mesostigmata). Florida State Museum Bulletin Biology Science, Gainesville, 5:267-302.

MUMA, M.H. 1962. New Phytoseiidae (Acarina: Mesostigmata). The Florida Entomologist, USA, 45, 1-10.

MUMA, M.H.; DENMARK, H.A. \& DELEON, D. 1970. Phytoseiidae of Florida. Arthropods of Florida and Neighboring land áreas, 6. Fla. Dept. Agr. Cons. Serv., Div. Plant Ind., Gainesville, 150p.

NESBITT, H.H.J. 1951. A taxonomic study of the Phytoseiidae (family Laelaptidae) predaceous upon Tetranychidae of economic importance. Zoologische Verhandelingen, The Netherlands, 12, 64 pp.+ 32 plates.

NORONHA, A.C.S.; CARVALHO, J.E.B.; CALDAS, R.C. 1997. Ácaros em citros nas condições de Tabuleiros Costeiros. Revista Brasileira de Fruticultura, Crus das Almas, V. 19, n. 3, p.373-376.

OATMANN, E.R.; GILSTRAP, F.E. \& VOTH, V.1976. Effect o different release rates of Phytoseiulus persimilis (Acarina: Phytoseiidae) on the twospotted spider mite on strawberry in southern California. Entomophaga, Paris, 21(3):269-273.

OATMANN, E.R. \& MCMURTRY, J.A.1966. Biological control of the twospotted mite on strawberry in southern California. Journal of Economic Entomology, Lanham, 59:433-439.

OATMANN, E.R.; MCMURTRY, J.A.; GILSTRAP, F.E. \& VOTH, V.1977a. Effect of releases of Amblyseius californicus, Phytoseiulus persimilis and Typhlodromus occidentalis on the twospotted spider mite on strawberry in southern California. Journal of Economic Entomology, Lanham, 70(1):45-47.

OATMANN, E.R.; MCMURTRY, J.A.; GILSTRAP, F.E. \& VOTH, V.1977b. Effect of releases of Amblyseius californicus on the twospotted spider mite on strawberry in southern California. Journal of Economic Entomology, Lanham, 70(1):638-640.

SCHICHA, E. \& ELSHAFIE, M. (1980). Four new species of phytoseiid mites from Australia, and three species from America redescribed (Acari: Phytoseiidae). Journal of the Australian Entomoligical Society, Australia, 19,27-36.

SCHUSTER, R.O. \& PRITCHARD, A.E. (1963). Phytoseiid mites of California. Hilgardia, USA, 34, 191-285.

SIMMONDS, S.P. 1971. Obervations on the possible control of Tetranychus urticae on strawberry by Phytoseiulus macropilis. Plant Pathology, 20:117-119.

STRONG, W.B. \& CROFT, B.A.1995. Inoculative release of phytoseiid mites into the rapidly expanding canopy of hop for control of Tetranychus urticae Koch. Environmental Entomology, Washington, 24:446-453.

Título: Ácaros predadores (Acari) associados à cultura do morango (Fragaria sp, Rosaceae) e plantas próximas no Estado do Rio Grande do Sul.

Autores: Ferla, NJ et al.

Biota Neotropica, Vol. 7 (número 2): 2007

http://www.biotaneotropica.org.br/v7n2/pt/abstract?article+b n01807022007

Recebido em 10/11/06 - Versão reformulada recebida em 29/03/07 - Publicado em 01/05/07

ISSN 1676-0603 